\title{
Robust Entanglement through Macroscopic Quantum Jumps
}

\author{
Jeremy Metz, Michael Trupke, and Almut Beige \\ Blackett Laboratory, Imperial College London, Prince Consort Road, London SW7 2BZ, United Kingdom
}

(Dated: August 27, 2018)

\begin{abstract}
We propose an entanglement generation scheme that requires neither the coherent evolution of a quantum system nor the detection of single photons. Instead, the desired state is heralded by a macroscopic quantum jump. Macroscopic quantum jumps manifest themselves as a random telegraph signal with long intervals of intense fluorescence (light periods) interrupted by the complete absence of photons (dark periods). Here we show that a system of two atoms trapped inside an optical cavity can be designed such that a dark period prepares the atoms in a maximally entangled ground state. Achieving fidelities above 0.9 is possible even when the single-atom cooperativity parameter $C$ is as low as 10 and when using a photon detector with an efficiency as low as $\eta=0.2$.

PACS numbers: 03.67.Mn, 03.67.Pp, 42.50.Lc
\end{abstract}

There are countless applications for highly entangled quantum states, ranging from the improvement of frequency standards [1] to quantum information processing, where they can be utilised for one-way quantum computing [2]. Consequently much effort has been made over the last few years to generate highly entangled states in the laboratory. For example, groups in Boulder and Innsbruck have entangled up to eight ions $[3$ and a four-photon cluster state has been created by Walther et al. 4]. However, scaling these setups to many more qubits is not straightforward. Adding qubits in ion traps increases the density of motional states and therefore requires some form of distributed quantum computing, possibly involving ion transport [5]. The main difficulties when entangling photons are the lack of an effective interaction and reliable photon storage.

Although not yet demonstrated experimentally, a promising alternative is to entangle atoms by coupling them via an optical cavity. The ability to position the atoms inside such a resonator to within a fraction of the optical wavelength [ 6 ] together with the possibility of effectively coupling distant cavities via optical networks [7, 8] promises a high degree of control and scalability. The quality of atom-cavity setups is often measured by the single-atom cooperativity parameter $C \equiv g^{2} / \kappa \Gamma$, which compares the atom-cavity coupling constant $g$ with the cavity photon decay rate $\kappa[9]$ and the atom decay rate $\Gamma$. After several years of experimentation with optical cavities values of $C$ of about 50 have been reported in the literature [10]. Significantly larger $C$ 's are currently only possible when using AlGaAs resonators with embedded quantum dots 11].

Despite recent progress, the achievable values of $C$ are still too low to allow for the high fidelity generation of highly entangled atomic states with existing proposals. Even when using dissipation-assisted stimulated Raman adiabatic passages (STIRAP) [12], strong detunings 13], or the idea of quantum computing using dissipation [14], a precision of more than 0.85 requires $C>100$. An exception is the scheme by Pachos and Walther [15] which

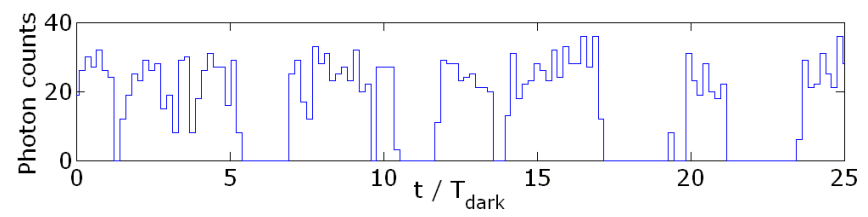

FIG. 1: Macroscopic quantum jumps in the fluorescence of two atoms trapped inside an optical cavity (c.f. Figure 2) obtained from the numerical simulation of a possible trajectory assuming $\Delta=50 \mathrm{~g}, \Gamma_{0}=\Gamma_{1}, \kappa=\Omega_{\mathrm{L}}=g$ and $\Gamma=\Omega_{\mathrm{M}}=0.1 \mathrm{~g}$, i.e. $C=10$. Shown is the number of photon emissions within time intervals of length $\Delta t=0.38 T_{\text {dark }}$ as a function of time.

achieves better results at the cost of a very slow and complex entangling STIRAP process. Even probabilistic schemes based on the detection of single photons promise fidelities above 0.9 only with perfect single photon detectors or require $C \gg 1$ [16. In contrast, we show here that it is possible to prepare two atoms in the maximally entangled ground state $\left|a_{01}\right\rangle \equiv(|01\rangle-|10\rangle) / \sqrt{2}$ with a fidelity $F$ above 0.9 even if $C$ is as low as 10 and when using a photon detector with an efficiency as low as $\eta=0.2$. Higher fidelities require a larger value of $C$ or $\eta$.

Achieving this is only possible, when dissipation plays a major role in the state preparation process. In the following we achieve this by employing a quantum mechanical phenomena known as quantum jumps. These abrupt transitions of the internal states of an atom, upon the emission or absorption of a light quantum, were proposed by Bohr as early as 1913 [17]. Bohr's quantum jumps were disturbing to many prominent physicists, including Schrödinger, as they raised many questions about our understanding of quantum mechanics [18]. However, it became possible to observe these jumps experimentally in the form of macroscopic light and dark periods, like the ones shown in Figure 1 In 1986, several group reported the blinking of the fluorescence of a single laser driven trapped ion [19].

In the language of modern quantum theory, macroscopic quantum jumps are a random telegraph process 


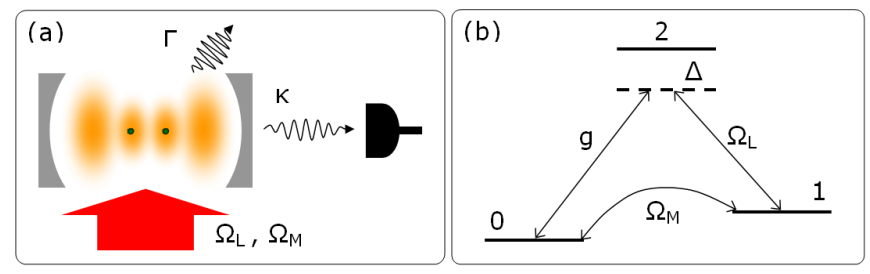

FIG. 2: (a) Experimental setup for the preparation of a maximally entangled state of two atoms trapped inside an optical cavity. (b) Level configuration of one atom with the qubit states $|0\rangle$ and $|1\rangle$.

with long intervals of intense photon emissions interrupted by periods of the complete absence of photons. They occur in the fluorescence of a single ion, if rapidly repeated measurements project the system either in a subspace of states, in which the systems emits photons at a high rate, or in a state, where it cannot undergo spontaneous emissions 20]. Dehmelt, who predicted the existence of macroscopic quantum jumps in 1975, rightly related this effect to electron shelving 21]. Small deviations from ideal measurements can cause a sudden change of the respective measurement outcome and are thereby responsible for transitions (jumps) from a light into a dark period and vice versa [22].

In the following, we generalise the setup introduced by Dehmelt and propose a scheme to entangle the electronic ground states of two atoms trapped inside an optical resonator. Interactions are applied such that the combined atom-cavity system generates macroscopic light and dark periods. Moreover, the desired state is the only dark state such that the absence of fluorescence indicates the shelving of the system with a high precision into the desired state. Turning off the applied laser fields within a dark period stops the system from returning into yet another light period and is enough to complete the state preparation.

The concrete experimental setup and the required atomic level diagram are shown in Figure 22 The trapping of the particles and the directions of the incoming laser fields should be chosen such that both atoms experience the same coupling constants. More concretely, the
0-2 transition of each atom couples to the cavity field with coupling strength $g$, while laser fields with Rabi frequency $\Omega_{\mathrm{M}}$ and $\Omega_{\mathrm{L}}$ drive the $0-1$ and the $1-2$ transitions, respectively. Here we are interested in the parameter regime where 23.

$$
\Omega_{\mathrm{M}}<g, \kappa, \Gamma, \Omega_{\mathrm{L}} \ll \Delta,
$$

with the detuning $\Delta$ as shown in Figure 2 and where $\Gamma$ denotes the spontaneous decay rate of level $2 . \Omega_{\mathrm{M}}$ can be realised using a microwave, a two-photon Raman transition via level 2 or a fourth level, since selection rules forbid the direct excitation of the $0-1$ transition.

In the following we model the atom-cavity system shown in Figure 2 by the master equation

$$
\dot{\rho}=-\frac{\mathrm{i}}{\hbar}\left[H_{\text {cond }} \rho-\rho H_{\text {cond }}^{\dagger}\right]+\mathcal{R}(\rho) .
$$

Here $\mathcal{R}$ is the reset operator describing the change of the system in the event of an emission and $H_{\text {cond }}$ is the conditional Hamiltonian relating to the no-photon evolution [22]. If $b$ is the annihilation operator for a photon inside the resonator, then $H_{\text {cond }}$ equals

$$
\begin{aligned}
H_{\text {cond }}= & \sum_{i=1,2}\left[\frac{1}{2} \hbar \Omega_{\mathrm{L}}|1\rangle_{i i}\left\langle 2\left|+\frac{1}{2} \hbar \Omega_{\mathrm{M}}\right| 0\right\rangle_{i i}\langle 1|+\right.\text { H.c. } \\
& \left.+\hbar g|0\rangle_{i i}\langle 2| b^{\dagger}+\text { H.c. }+\hbar\left(\Delta-\frac{i}{2} \Gamma\right)|2\rangle_{i i}\langle 2|\right] \\
& -\frac{i}{2} \hbar \kappa b^{\dagger} b
\end{aligned}
$$

in the interaction picture with respect to the interactionfree Hamiltonian and within the rotating wave approximation. Moreover $\mathcal{R}(\rho)$ is given by

$$
\mathcal{R}(\rho)=\sum_{j=0,1} \sum_{i=1,2} \Gamma_{j}|j\rangle_{i i}\langle 2|\rho| 2\rangle_{i i}\langle j|+\kappa b \rho b^{\dagger}
$$

with $\Gamma_{j}$ being the spontaneous decay rate of the $2-j$ transition $\left(\Gamma=\Gamma_{0}+\Gamma_{1}\right)$.

As the operators (3) and (4) treat both atoms in exactly the same way, it is convenient to introduce the states $\left|s_{j k}\right\rangle \equiv(|j k\rangle+|k j\rangle) / \sqrt{2}$ and $\left|a_{j k}\right\rangle \equiv(|j k\rangle-$ $|k j\rangle) / \sqrt{2}$. Using this notation, Eq. (3) becomes

$$
\begin{aligned}
H_{\text {cond }}= & \frac{1}{2} \hbar \Omega_{\mathrm{L}}\left[\left|s_{01}\right\rangle\left\langle s_{02}|+| a_{01}\right\rangle\left\langle a_{02}\right|+\sqrt{2}\left(|11\rangle\left\langle s_{12}|+| s_{12}\right\rangle\langle 22|\right)+\text { H.c. }\right]+\frac{1}{2} \hbar \Omega_{\mathrm{M}}\left[\left|s_{02}\right\rangle\left\langle s_{12}|+| a_{02}\right\rangle\left\langle a_{12}\right|\right. \\
& \left.+\sqrt{2}\left(|00\rangle\left\langle s_{01}|+| s_{01}\right\rangle\langle 11|\right)+\text { H.c. }\right]+\hbar g\left[\left|s_{01}\right\rangle\left\langle s_{12}\left|b^{\dagger}-\right| a_{01}\right\rangle\left\langle a_{12}\right| b^{\dagger}+\sqrt{2}\left(|00\rangle\left\langle s_{02}|+| s_{02}\right\rangle\langle 22|\right) b^{\dagger}+\text { H.c. }\right] \\
& -\frac{\mathrm{i}}{2} \hbar \kappa b^{\dagger} b+\hbar\left(\Delta-\frac{\mathrm{i}}{2} \Gamma\right)\left[\left|s_{02}\right\rangle\left\langle s_{02}|+| a_{02}\right\rangle\left\langle a_{02}|+| s_{12}\right\rangle\left\langle s_{12}|+| a_{12}\right\rangle\left\langle a_{12}|+2| 22\right\rangle\langle 22|\right]
\end{aligned}
$$

Similarly, Eq. (4) can be written as

$$
\mathcal{R}(\rho)=\sum_{j=0,1} \sum_{i=1,2} \Gamma_{j} R_{j i} \rho R_{j i}^{\dagger}+\kappa b \rho b^{\dagger}
$$

with the reset operators

$$
\begin{aligned}
& R_{01} \equiv|00\rangle\left\langle s_{02}\left|+\frac{1}{\sqrt{2}}\left(\left|s_{01}\right\rangle\left\langle s_{12}|-| a_{01}\right\rangle\left\langle a_{12}\right|\right)+\right| s_{02}\right\rangle\langle 22|, \\
& R_{02} \equiv|00\rangle\left\langle a_{02}\left|+\frac{1}{\sqrt{2}}\left(\left|s_{01}\right\rangle\left\langle a_{12}|-| a_{01}\right\rangle\left\langle s_{12}\right|\right)-\right| a_{02}\right\rangle\langle 22|, \\
& R_{11} \equiv|11\rangle\left\langle s_{12}\left|+\frac{1}{\sqrt{2}}\left(\left|s_{01}\right\rangle\left\langle s_{02}|+| a_{01}\right\rangle\left\langle a_{02}\right|\right)+\right| s_{12}\right\rangle\langle 22|, \\
& R_{12} \equiv|11\rangle\left\langle a_{12}\left|+\frac{1}{\sqrt{2}}\left(\left|s_{01}\right\rangle\left\langle a_{02}|+| a_{01}\right\rangle\left\langle s_{02}\right|\right)-\right| a_{12}\right\rangle\langle 22| .
\end{aligned}
$$




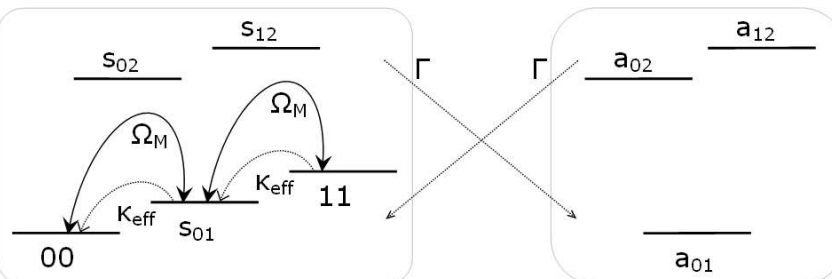

FIG. 3: Effective evolution within a light and a dark period involving the states $|00\rangle,\left|s_{01}\right\rangle,|11\rangle$ and $\left|a_{01}\right\rangle$, respectively. Transitions between the subspaces can occur after an emission from the excited atomic states $\left|s_{02}\right\rangle,\left|s_{12}\right\rangle$ and $\left|a_{02}\right\rangle$.

Given the parameter regime (10), the excited atomic states with population in $|2\rangle$ evolve much faster than all other states and can be adiabatically eliminated. Moreover, coherent transitions between states with different numbers of photons in the cavity take place with the frequency $g_{\text {eff }} \equiv-\Omega_{\mathrm{L}} g /(\sqrt{2} \Delta) \ll \kappa$. We can therefore also eliminate the states with more than one photon in the resonator. Doing so and denoting the number of cavity photons by $n$, while $\alpha_{j k, n}, \sigma_{j k, n}$ and $\xi_{j j, n}$ are the amplitudes of the states $\left|a_{j k}, n\right\rangle,\left|s_{j k}, n\right\rangle$ and $|j j, n\rangle$, respectively, we find that

$$
\begin{aligned}
& \alpha_{02,0}=-\left(\Omega_{\mathrm{L}} / 2 \Delta\right) \alpha_{01,0}, \alpha_{12,0}=\xi_{22,0}=0, \\
& \sigma_{02,0}=-\left(\Omega_{\mathrm{L}} / 2 \Delta\right) \sigma_{01,0}, \sigma_{12,0}=\left(\Omega_{\mathrm{L}} / \sqrt{2} \Delta\right) \xi_{11,0}, \\
& \xi_{00,1}=-\left(2 \mathrm{i} g_{\mathrm{eff}} / \kappa\right) \sigma_{01,0}, \sigma_{01,1}=-\left(2 \mathrm{i} g_{\mathrm{eff}} / \kappa\right) \xi_{11,0}
\end{aligned}
$$

up to first order in $1 / \Delta$. Effectively the system can be described by

$$
\begin{aligned}
H_{\text {cond }}= & \frac{1}{\sqrt{2}} \hbar \Omega_{\mathrm{M}}\left[|00,0\rangle\left\langle s_{01}, 0|+| s_{01}, 0\right\rangle\langle 11,0|+\text { H.c. }\right] \\
& +\hbar \Delta_{\mathrm{L}}[|11,0\rangle\langle 11,0|-| 00,0\rangle\langle 00,0|] \\
& -\frac{i}{2} \hbar \kappa_{\mathrm{eff}}\left[\left|s_{01}, 0\right\rangle\left\langle s_{01}, 0|+| 11,0\right\rangle\langle 11,0|\right] \\
\mathcal{R}(\rho)= & \kappa_{\text {eff }}\left[|00,0\rangle\left\langle s_{01}, 0|+| s_{01}, 0\right\rangle\langle 11,0|\right]
\end{aligned}
$$

with $\Delta_{\mathrm{L}} \equiv-\Omega_{\mathrm{L}}^{2} /(4 \Delta)$ and $\kappa_{\text {eff }} \equiv 4 g_{\text {eff }}^{2} / \kappa$.

The dark state of a system is the state with a negligible spontaneous decay rate. Furthermore, the system's evolution should not be able to transfer this state into one that can cause an emission. From Eqs. (9), we see that this applies only to the zero eigenstate $\left|a_{01}, 0\right\rangle$ of $H_{\text {cond. }}$ From the above calculations we also see that a light period mainly involves the states $|00,0\rangle,\left|s_{01}, 0\right\rangle$ and $|11,0\rangle$. An interaction drives these states with frequency $\Omega_{\mathrm{M}}$ and detuning $\Delta_{\mathrm{L}}$ continuously, as shown in Figure 3 Population in $\left|s_{01}, 0\right\rangle$ and $|11,0\rangle$ can cause a photon to leak out through the cavity mirrors with the effective rate $\kappa_{\text {eff }}$. After a certain time, these processes result in a stationary state. Using Eq. (9) and setting $\dot{\rho}=0$ we find the steady state populations

$$
\begin{aligned}
P_{00,0} & =1-P_{s_{01}, 0}-P_{11,0} \\
P_{s_{01}, 0} & =\left(1+8 x^{2}\right) /\left(3+16 x^{2}+16 x^{4}\right), \\
P_{11,0} & =1 /\left(3+16 x^{2}+16 x^{4}\right)
\end{aligned}
$$

with $x \equiv-\Omega_{\mathrm{L}}^{2} / 4 \Delta \Omega_{\mathrm{M}}$. From Eq. (8) we see that there is also a small population in the states $|00,1\rangle$ and $\left|s_{01}, 1\right\rangle$ with one photon in the cavity. One therefore has $\langle n\rangle=$ $\left(\kappa_{\text {eff }} / \kappa\right)\left(P_{s_{01}, 0}+P_{11,0}\right)$ which implies

$$
T_{\text {cav }}=\left(3+4 x^{2}\right) \cdot \frac{\kappa \Delta^{2}}{4 g^{2} \Omega_{\mathrm{L}}^{2}}
$$

for the mean time between two cavity photon emissions, since $T_{\text {cav }}=1 / \kappa\langle n\rangle$.

Transitions between light and dark periods occur since population in the states $\left|s_{01}, 0\right\rangle,|11,0\rangle$ and $\left|a_{01}, 0\right\rangle$ results in small amounts of population in $\left|s_{02}, 0\right\rangle,\left|s_{12}, 0\right\rangle$, and $\left|a_{02}, 0\right\rangle$, respectively (c.f. Eq. (8)). This eventually leads to an atomic decay from level 2 which prepares the setup in a superposition of a light and the dark state. Now there are three possibilities: (i) After a short time the system emits another photon via an atomic decay and returns into a neither symmetric nor antisymmetric state, which brings us back to the initial situation. (ii) No photon is emitted for a time, which is long compared to $T_{\text {cav }}$, preparing the atoms in $\left|a_{01}\right\rangle$. (iii) A photon leaks out through the cavity mirrors and prepares the setup in a symmetric state (c.f. Eq. (92)) with no population in the dark state. In the latter two cases a transition between a light and a dark period may have occurred. The reason for the projection into one of the subspaces is that the observation of cavity leakage reveals information about the system. This measurement projects the atoms either into a symmetric state or into $\left|a_{01}\right\rangle$.

More concretely, the end of a dark period is caused by a spontaneous emission from the state $\left|a_{02}, 0\right\rangle$. Its population within a dark period can be calculated using Eq. (8) with $\alpha_{01,0}=1$. Moreover we see from Eq. (7) that such an emission transfers the atoms with rate $\Gamma_{0}$ into the state $|00\rangle$ and with rate $\frac{1}{2} \Gamma_{1}$ into $\left|s_{01}\right\rangle$. Both together imply

$$
T_{\text {dark }}=\frac{1}{2 \Gamma_{0}+\Gamma_{1}} \cdot \frac{8 \Delta^{2}}{\Omega_{\mathrm{L}}^{2}} .
$$

Similarly, taking Eqs. (8) and (10) into account, we see that a light period ends at any time with the probability density $\frac{1}{2} \Gamma_{0} P_{s_{02}, 0}+\frac{1}{2} \Gamma_{1} P_{s_{12}, 0}$ which yields

$$
T_{\text {light }}=\frac{3+16 x^{2}+16 x^{4}}{2 \Gamma_{0}+\left(1+8 x^{2}\right) \Gamma_{1}} \cdot \frac{8 \Delta^{2}}{\Omega_{\mathrm{L}}^{2}}
$$

for the mean length of a light period.

Crucial for the ability to distinguish a light from a dark period is that $T_{\text {dark }}$ is sufficiently longer than $T_{\text {cav }}$. For a wide range of parameters, namely for $\Omega_{\mathrm{L}}^{4} \ll\left(4 \Delta \Omega_{\mathrm{M}}\right)^{2}$, one has $x^{2} \ll 1$ and the ratio $T_{\text {dark }} / T_{\text {cav }}$ is to a very good approximation given by its maximum $32 g^{2} / 3 \kappa\left(2 \Gamma_{0}+\Gamma_{1}\right)$. Therefore, the obtainable telegraph signal (c.f. Figure 1) becomes clearer the larger the single atom cooperativity parameter $C$. However, the proposed state preparation 


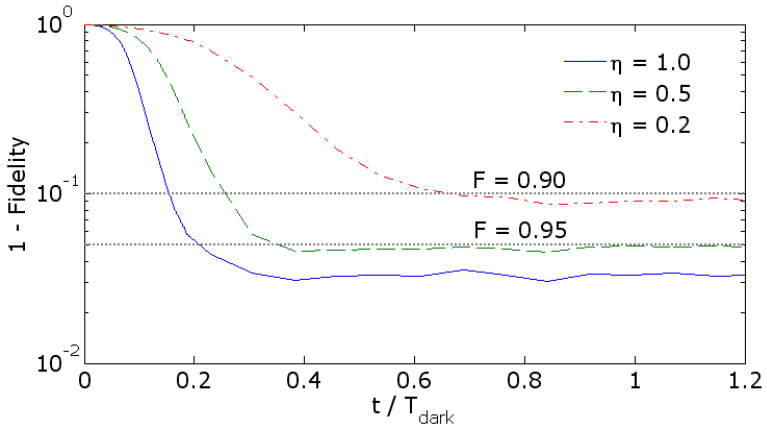

FIG. 4: The deviation from unity of the fidelity $F$ of the state prepared after the detection of no photon for a time $t$ for different detector efficiencies $\eta$ and for the same parameters as in Figure $(C=10)$ obtained from a numerical simulation.

scheme, namely turning off the laser fields upon the detection of no photon for a time $t$ of the order of $T_{\text {dark }}$, also works for relatively small values of $C$. For $C=10$ a dark period is on average 70 times longer than $T_{\text {cav }}$ and the entangled state $\left|a_{01}\right\rangle$ can be obtained with a fidelity above 0.9 for $t>0.7 T_{\text {dark }}$ even when using a photon detector

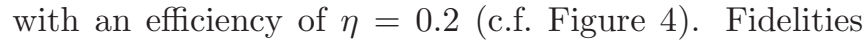
above 0.95 require $\eta \geq 0.5$ and $t>0.4 T_{\text {dark. Generally }}$ it does not take long to find the system in a dark period, since $T_{\text {light }}$ is on average only about three times longer than $T_{\text {dark }}$.

In summary, we have described a scheme that can be used to entangle two atoms trapped inside an optical cavity. The generalisation of our scheme to a wider class of entangled states, including cluster states for one-way quantum computing [2], is straightforward. This can be achieved by placing more than two atoms simultaneously into the cavity and by moving them subsequently in and out of the resonator 24]. Problems might arise from having to trap the atoms at fixed positions with respect to the cavity mode and the incoming laser fields. However, the techniques for doing this have improved significantly over recent years [ [] . In addition, the cavity can be used to cool the atomic motion between different attempts to entangle the atoms, as recently demonstrated by Nußmann et al. 25.

Characteristic for the proposed scheme is its constructive use of dissipation. Indeed it is well known that dissipation causes irreversibility and reduces the entropy of coupled quantum systems. Applications are the cooling of single atoms to very low temperatures [26], the fusion of Bose Einstein condensates 27] and measurementbased quantum computing schemes 2, 8, 14, 15. Moreover, the presence of spontaneous decay rates can be used to stabilise the time evolution of a system [12. In contrast to this, the present paper describes a dissipative process, which transfers the system into a macroscopic dark period. The successful state preparation is heralded by the interruption of a long interval of intense fluorescence. It is therefore possible to entangle atoms with a high fidelity even when using realistic photon detectors and a relatively modest atom-cavity setup. Because of their reliability, we believe that macroscopic quantum jumps will become a very useful tool for the controlled generation of entanglement in physical systems.

Acknowledgment. We thank E. Hinds, P. L. Knight, Y. L. Lim, D. Meschede's group and A. Serafini for helpful discussions. A. B. acknowledges support from the Royal Society and the GCHQ. This work was supported in part by the EU network SCALA and the UK EPSRC through the QIP IRC.

[1] J. J. Bollinger et al., Phys. Rev. A 54, R4649 (1996).

[2] R. Raussendorf and H. J. Briegel, Phys. Rev. Lett. 86, 5188 (2001).

[3] D. Leibfried et al., Nature 438, 639 (2005); H. Häffner et al., Nature 438, 643 (2005).

[4] P. Walther et al., Nature 434, 169 (2005).

[5] D. Kielpinski et al., Nature 417, 709 (2002).

[6] S. Nußmann et al., Phys. Rev. Lett. 95, 173602 (2005); M. Trupke et al., Appl. Phys. Lett. 87, 211106 (2005); Y. Miroshnychenko et al., quant-ph/0606113

[7] B. Kraus and J. I. Cirac, Phys. Rev. Lett. 92, 013602 (2004).

[8] Y. L. Lim et al., Phys. Rev. Lett 95, 030505 (2005).

[9] Note that many experimental papers use this notation for the cavity field decay rate, which equals $\frac{1}{2} \kappa$.

[10] J. Ye et al., Phys. Rev. Lett. 83, 4987 (1999); J. A. Sauer et al., Phys. Rev. A 69, 051804(R) (2004); P. Maunz et al., Phys. Rev. Lett. 94, 033002 (2005).

[11] K. Srinivasan et al., Appl. Phys. Lett. 86, 151106 (2005).

[12] T. Pellizzari et al., Phys. Rev. Lett. 75, 3788 (1995); C. Marr et al., Phys. Rev. A 68, 033817 (2003).

[13] S.-B. Zheng and G. C. Guo, Phys. Rev. Lett. 85, 2392 (2000); X. X. Yi et al., Phys. Rev. Lett. 90, 097902 (2003).

[14] A. Beige et al., Phys. Rev. Lett. 85, 1762 (2000).

[15] J. Pachos and H. Walther, Phys. Rev. Lett. 89, 187903 (2002).

[16] C. Cabrillo et al., Phys. Rev. A 59, 1025 (1999); M. B. Plenio et al., Phys. Rev. A 59, 2468 (1999); A. S. Sørensen and K. Mølmer, Phys. Rev. Lett. 91, 097905 (2003); P. Lougovski et al., Phys. Rev. A 71, 013811 (2005); D. E. Browne et al., Phys. Rev. Lett. 91, 067901 (2003).

[17] N. Bohr, Philos. Mag. 26, 476 (1913).

[18] For a review see R. Blatt and P. Zoller, Eur. J. Phys. 9, 250 (1988).

[19] W. Nagourney et al., Phys. Rev. Lett. 56, 2797 (1986); Th. Sauter et al., Phys. Rev. Lett. 57, 1696 (1986); J. C. Bergquist et al., Phys. Rev. Lett. 57, 1699 (1986).

[20] R. J. Cook and H. J. Kimble, Phys. Rev. Lett. 54, 1023 (1985); J. Javanainen, Phys. Rev. A 33, R2121 (1986); D. T. Pegg et al., Phys. Rev. A 33, 4085 (1986).

[21] H. G. Dehmelt, Bull. Am. Phys. Soc. 20, 60 (1975).

[22] A. Beige and G. C. Hegerfeldt, J. Phys. A 30, 1323 (1997); G. C. Hegerfeldt, Fortschr. Phys. 46, 595 (1998).

[23] The condition $\Omega_{\mathrm{M}}<g, \kappa, \Gamma, \Omega_{\mathrm{L}}$ is not crucial for the scheme to work but simplifies the following calculations. 
[24] J. Metz et al., (in preparation).

[25] S. Nußmann et al., Nature Physics 1, 122 (2005).

[26] D. J. Wineland and H. G. Dehmelt, Bull. Am. Phys. Soc.
20, 637 (1975).

[27] D. Jaksch et al., Phys. Rev. Lett. 86, 4733 (2001). 